\title{
The Effect of Sport-Specific High Intensity Interval Training on Ghrelin Levels and Body Composition in Youth Wrestlers
}

\author{
${ }^{1}$ Keivan Akhoundnia, ${ }^{2}$ Amir Rashid Lamir ${ }^{*},{ }^{1}$ Rambod Khajeie, ${ }^{3}$ Hamid Arazi \\ ${ }^{1}$ Department of Physical Education, Neyshabur Branch, Islamic Azad University, Neyshabur, Iran. \\ ${ }^{2}$ Department of Exercise Physiology, Faculty of Sport Science, Ferdowsi University of Mashhad, \\ Mashhad, Iran. ${ }^{3}$ Department of Exercise Physiology, Faculty of Sport Sciences, University of Guilan, \\ Rasht, Iran.
}

Submitted 08 August 2018; Accepted in final form 27 October 2018.

\begin{abstract}
Background. High-Intensity Interval Training (HIIT) may affect Acylated Ghrelin (AG) through changes in body composition. Objectives. The present study was designed to investigate and compare the effects of two different orders of wrestling-based HIIT on AG levels and body composition in highly trained wrestlers. Methods. Forty-two male wrestlers (aged 16-21 years) were randomly assigned to one of three groups: HIIT23 (2 sets of 3 min wrestling techniques; by $80-90 \% \mathrm{HR}_{\max }$ ); HIIT32 (3 sets of 2 min wrestling techniques; by $90-100 \% \mathrm{HR}_{\max }$ ); and a control group that performed routine wrestling exercises. HIIT protocol consisted of eight techniques which were performed three days/week for eight weeks. Body composition and total plasma AG were measured $48 \mathrm{~h}$ before and after the protocol. Results. Body fat percentage $(p<0.01)$ and weight $(p<0.05)$ were significantly decreased in the HIIT groups from preto post-test. There was also a significant decrease in body fat percentage of both HIIT groups compared to the control group ( $p=0.002$ ). Although a significant increase in post-test AG was observed in all the groups, AG levels in HIIT groups were approximately twofold higher than control group $(p<0.01)$. Conclusion. It seems that eight weeks of wrestling-based HIIT can cause to significant decreases in body fat percentage and weight which is related to increase in total levels of plasma AG in highly trained wrestlers.
\end{abstract}

\section{KEY WORDS: Acylated Ghrelin, HIIT, Wrestling Techniques, Body Fat Percentage, Body Weight.}

\section{INTRODUCTION}

Wrestling is a weight class sport and maintaining a specific body weight is important for wrestlers. Energy balance which means the balance between energy intake and its expenditure is the fundamental principle that is underlying body weight regulation (1). Thus, the formerly mentioned components of energy balance must be targeted for maintaining a desired body weight. If the balance is not maintained by wrestlers, their body weight is changed and they must achieve a target weight for competitions which may have a negative impact on their performance. Clearly, exercise protocols that simultaneously target both components of energy balance in a manner are needed for improvements in body composition. Therefore, exercises are highly desirable which sufficiently increase energy expenditure without promoting subsequent increases in energy intake or appetite. (2).

High-Intensity Interval Training (HIIT) involves short bouts of exercise with the intensity between 80 to $100 \% \mathrm{VO}_{2 \max }$ or maximum heart

*. Corresponding Author:

Amir Rashid Lamir, Associate Professor,

Zip Code: 9177948979, Faculty of Sport Science, Ferdowsi University of Mashhad, Azadi Square, Mashhad, Iran.

E-mail: rashidlamir@um.ac.ir 
rate $\left(\mathrm{HR}_{\max }\right)$ interspersed with periods of complete rest for recovery, active rest or lowintensity effort (3). Recent findings suggest that near maximal exercise (more than $75 \% \mathrm{VO}_{2 \max }$ or $\mathrm{HR}_{\max }$ ) may suppress hunger through alterations in appetite-regulating hormones, which subsequently reduces energy intake $(2,4-7)$. Lowvolume HIIT induces significant improvements in body composition via significant reductions in fat mass (7-9). The observed decreases in fat mass have been attributed to both increased exerciseinduced energy expenditure $(7,10,11)$ and decreased post-exercise energy intake $(5,6,12)$, potentially through appetite suppression (13). Because of the brief nature of HIIT, improvements in body composition may also be attributed to changes in the appetite-regulating hormones in a manner that contributes to a decrease in energy intake after exercise.

Ghrelin, known as the only peripheral appetite-regulating hormone, is a 28 amino acid peptide hormone which has been produced predominantly by a kind of specialized cells in the stomach (14). Ghrelin has a potential role in appetite and its administration augments food intake and subsequently leads to gain in body weight $(7,14)$. It has been reported that acute HIIT resulted in a significant suppression of appetite and Acylated Ghrelin (AG) $(5,13,15)$. Martins et al., (15) found an acute bout of HIIT which leads to a suppression of AG with no compensatory increases in appetite. A greater suppression of appetite and AG has also been reported following more intense HIIT (30 s "allout" cycling x 6 bouts, 4 min recovery) than moderate-intensity continuous training (MICT; 60 min cycling at $\left.65 \% \mathrm{VO}_{2 \max }\right)(13)$. The majority of research is examining the effects of HIIT on appetite-regulating hormones that have involved acute responses in obese or overweight individuals. However, one study revealed that chronic HIIT ( 3 times/week for 12 weeks) has no significant effect on plasma concentration of AG in sedentary obese women and men (16). Similar results have also been reported in another study that compared appetite regulation in overweight inactive men following 12 weeks of HIIT (alternating $15 \mathrm{~s}$ cycling at $170 \% \mathrm{VO}_{2 \text { peak }}$ and $60 \mathrm{~s}$ at $\left.34 \% \mathrm{VO}_{2 \text { peak }}\right)$ and MICT $\left(60 \% \mathrm{VO}_{2 \text { peak }}\right)(17)$. Given the limited number of studies are examining the chronic effect of HIIT on AG and body composition in weight class athletes, additional research is required. Therefore, we aimed to compare the eight weeks effect of two types of wrestling-based HIIT on body composition and plasma levels of $\mathrm{AG}$ in elite wrestlers.

\section{MATERIALS AND METHODS}

Participants. Following the approval of Nishabur Islamic Azad University's Ethics Committee, forty-two highly trained male wrestlers (age, 17.88 $\pm 1.23 \mathrm{yr}$ ) participated in the study. They all have had medal winners in provincial or national level championships. All participants had trained, on average, three times per week during the 4 weeks prior to the study. Exclusion criteria were smoking, a history of medical conditions and/or eating disorders which have been known to affect appetite, taking any dietary supplements or medication which has been known to affect appetite or induce weight loss at the time of the study. The purpose of the investigation and experimental procedures were fully explained to the participants and all signed a written consent before enrolling in the study.

Study Design. This was a randomized design study that participants were randomly allocated to either HIIT23 $(n=15)$, HIIT32 $(n=15)$, or control group $(n=12)$ for 8 weeks. All participants completed two familiarization/pilot sessions (one week) prior to the first training session to introduce HIIT protocols, suitable exercise intensity (a percentage of $\mathrm{HR}_{\max }$; automatic $\mathrm{HR}$ monitor; Beurer; PM80; Germany) and reduce any learning effects during subsequent sessions. Preliminary anthropometric measurements including height, body weight, Body Mass Index (BMI), body fat percentage (skinfold thickness; Lafayette Instrument Co, 01127A, USA), and Lean Body Mass [LBM, body weight (kg) skinfold body fat $(\mathrm{kg})]$ were obtained during the second familiarization session. Blood was sampled in the fasted state $48 \mathrm{~h}$ before and after the training protocol. Participants also completed a self-recorded food diary in the $24 \mathrm{~h}$ prior to preand post-test measurements and they were asked to maintain their habitual diet during the study period.

Exercise Intervention. The exercise protocols were carried out with a suitable interval from the next wrestling competitions. Both exercise training groups (HIIT23 and HIIT32) underwent an 8-week supervised wrestling-based HIIT programme, three sessions per week. Each exercise session consisted of 10 
min of warm-up (jogging and running followed by static and dynamic stretching of the lower and upper limbs), $20 \mathrm{~min}$ of routine wrestling exercises, 22-55 min of HIIT (eight wrestling techniques), and $10 \mathrm{~min}$ of cool-down (standardized stretching). Routine wrestling exercises and the wrestling-based HIIT were interchanged alternately in the exercise sessions. Participants who allocated to HIIT23 exercised 2 sets of 3 min wrestling techniques at 80 to $90 \%$ $\mathrm{HR}_{\max }$; while participants who randomized to HIIT32 were required to complete 3 sets of 2 min wrestling techniques with 90 to $100 \% \mathrm{HR}_{\max }$. Both HIIT23 and HIIT32 groups completed 2 bouts of HIIT during weeks 1-2 and one bout was added to the protocol every 2 weeks. Thus, the duration of HIIT progressed as follows: weeks 1-2 (22 $\mathrm{min})$, weeks 3-4 (33 $\mathrm{min})$, weeks 5-6 (44 $\mathrm{min})$, and weeks 7-8 (55 $\mathrm{min})$. The rest intervals of the HIIT32 group were equal to 120 and $180 \mathrm{~s}$ between sets and bouts, respectively. Participants in the HIIT23 group rested equal to 90 and $120 \mathrm{~s}$ between sets and bouts, respectively (18). The wrestling techniques which have been consisted of single leg takedown, cross-buttocks, double leg takedown, flying mare, single leg takedown in opposite position, over under throw, double leg pickup, and standing spin under that were performed with a 5-meter distance from each other. Each participant was required to complete the first technique and then to perform other seven techniques quickly. This was repeated until the end of the time of each set. It should be noted that the control group performed 65-95 min of routine wrestling exercises between 55 to $65 \%$ $\mathrm{HR}_{\max }$ with the same timing and frequency during the study period (18).

Blood Sampling and Biochemical Analysis. Participants attended the laboratory following an overnight fast (10-12 h) and rested on a couch for $10 \mathrm{~min}$. Blood samples $(10 \mathrm{ml})$ were then collected from the antecubital vein into plastic tubes containing Ethylene Diamine Tetra-acetic Acid (EDTA) and Para Hydroxy Mercury Benzoic-acid (PHMB). All samples were centrifuged at $3000 \mathrm{~g}$ for $10 \mathrm{~min}$ and obtained plasma was stored frozen at $-20 \mathrm{C}$ for subsequent analysis.

Total plasma AG was quantified by commercial Radio Immune Assay (RIA) method using human-specific ELISA kits (CUSABIO BIOTECH, Wuhan, China) and according to the manufacturer's instructions. The sensitivity of the assays was equal to $0.16 \mathrm{pg} / \mathrm{ml}$ and the intra-assay coefficient of variation was by $7.9 \%$. All samples of each participant were run in duplicate and in the same assay.

Statistical Analysis. Data was analyzed using SPSS software version 18.0 for Windows (SPSS Inc., Chicago, IL, USA). Means and Standard Deviation (SD) were calculated and the level of statistical significance was set at $P<0.05$. All data was tested for normality using the Shapiro-Wilk test before subsequent statistical analysis. A paired t-test was conducted to evaluate within-group differences. A univariate ANOVA was used to compare the difference of plasma AG levels and body composition between the three groups. Pretest levels of AG, age and BMI were entered as covariates and post hoc comparisons were carried out using Bonferroni test where appropriate.

\section{RESULTS}

No significant differences between groups existed in energy intake (table 1) and initial body weight, BMI, and body fat percentage. However, pre-test levels of AG were significantly higher in the HIIT32 group compared to the control group ( $p=0.020$ ). Paired t-tests showed that post-test body weight and body fat percentage were significantly decreased in both HIIT23 (body weight $=1.42 \pm$ $1.92 \%, p=0.008$; body fat percentage $=8.92 \pm$ $8.32 \%, \quad p=0.002$ ) and HIIT32 (body weight $=1.22 \pm 2.34 \%, \quad p=0.044$; body fat percentage $=13.25 \pm 6.8 \%, p<0.01$ ) groups, but BMI was decreased only in the HIIT23 group $(p=0.010)$. There were no differences in LBM from pre- to post-test in all the groups. It was also revealed that the variables of body composition did not change in the control group.

Between-group comparisons showed that post-test body fat percentage of both HIIT23 (mean difference $=4.19, p=0.002$ ) and HIIT32 (mean difference $=4.21, p=0.002$ ) groups were significantly lower than the control group (Figures 1-A to 1-D).

As shown in figure 4, plasma AG concentrations were significantly increased in all the groups (HIIT23 = $6.94 \pm 3.12 \%$; HIIT32 = 6.96 $\pm 3.55 \%$; control group $=2.75 \pm 0.92 \% ; p<0.01)$. After adjustment for pre-test AG, univariate ANOVA test revealed that post-test plasma AG levels of the HIIT23 (mean difference $=5.19, p<0.01$ ) and HIIT32 (mean difference $=5.57, p<0.01$ ) groups were significantly higher than the control group. 
Table 1. Mean $( \pm$ SE) of energy intake before (pre-test) and after (post-test) 8 weeks of HIIT

\begin{tabular}{|c|c|c|c|c|}
\hline Groups & & Energy intake (Cal) & within-group significance & between-group significance \\
\hline \multirow{2}{*}{ HIIT23 $(n=15)$} & Pre-test & $3178.10 \pm 300.55$ & \multirow{2}{*}{0.101} & \multirow{6}{*}{0.282} \\
\hline & Post-test & $3268.63 \pm 275.95$ & & \\
\hline \multirow{2}{*}{ HIIT32 $(n=15)$} & Pre-test & $3303.93 \pm 384.75$ & \multirow{2}{*}{0.822} & \\
\hline & Post-test & $3292.36 \pm 344.93$ & & \\
\hline \multirow{2}{*}{ Control $(n=12)$} & Pre-test & $3211.92 \pm 315.65$ & \multirow{2}{*}{0.675} & \\
\hline & Post-test & $3184.29 \pm 268.38$ & & \\
\hline
\end{tabular}

HIIT23: (high-intensity interval training, 2 sets of 3 min exercises + routine wrestling exercises),

HIIT32: (high-intensity interval training, 3 sets of 2 min exercises + routine wrestling exercises),

Control: (routine wrestling exercises).

A

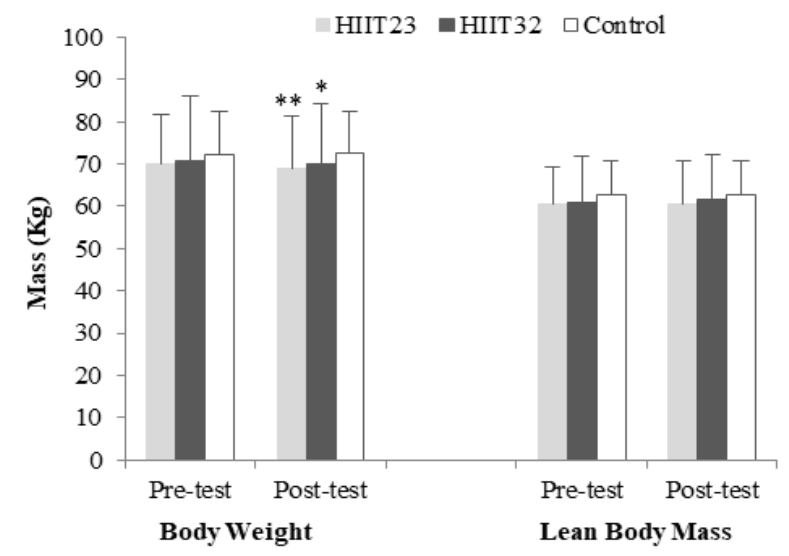

C

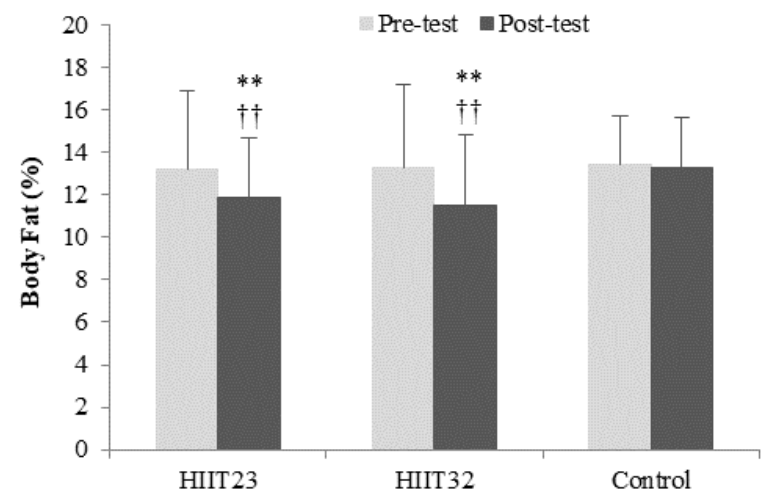

B

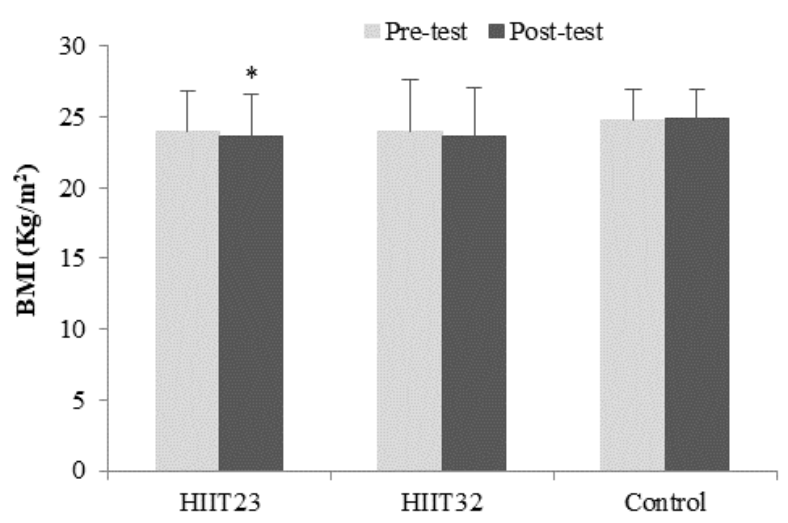

D

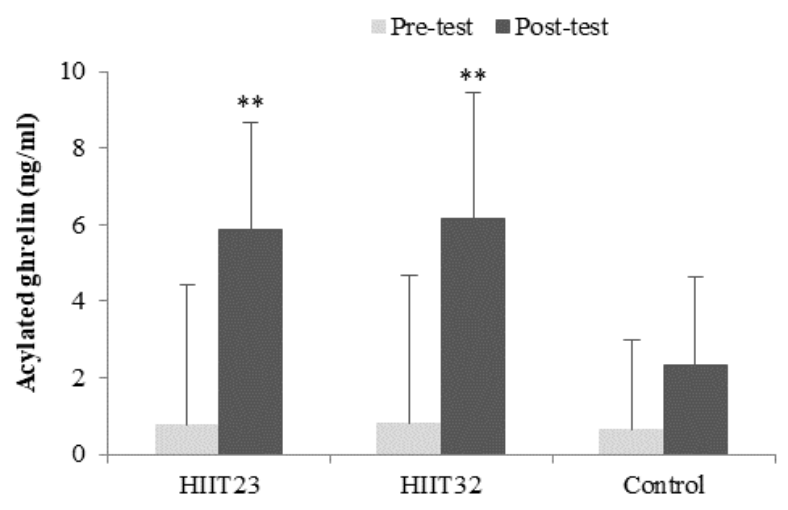

Figure 1. Changes in (A) body weight and lean body mass, (B) body mass index (BMI), (C) body fat percentage, and (D) acylated ghrelin from pre- to post-test within and between groups. HIIT23: high-intensity interval training ( 2 sets of 3 min exercises + routine wrestling exercises); HIIT32: high-intensity interval training (3 sets of 2 min exercises + routine wrestling exercises); Control: routine wrestling exercises. *: p<0.05 Post-test vs Pre-test, $\dagger \dagger:$ p $<0.01$ Post-test vs Pre-test, **: p<0.01 vs control.

\section{DISCUSSION}

To our knowledge, this was one of the few studies, which is investigating the impact of specific HIIT in trained athletes. The main aim of the present study was to compare the effects of two different orders of wrestling-based HIIT which have been added to the routine wrestling exercises with an equivalent period of routine 
wrestling exercises on body composition and plasma concentrations of AG in wrestlers. There were no statistically significant differences in total energy intake, $24 \mathrm{~h}$ before the study measurements. We found significant decreases in post-test body fat percentage and weight in the HIIT groups. Our findings also can be more important when compared with the control group. This suggests that HIIT which is designed on the basis of the specific sport techniques may be considered as a meaningful and important strategy for weight management in athletes. Most studies which are questioning the effect of HIIT programs on body composition have been conducted on overweight or obese individuals (19-23). Generally, it has been shown that shortterm HIIT elicit modest improvements in body fat levels in overweight and obese adults (19). More recently, Khammassi et al. (20) conducted an HIIE program for 12 weeks with three weekly 25minute HIIE sessions in healthy untrained overweight/obese males. HIIT consisted of a 30second running which has been followed by $30 \mathrm{~s}$ of active recovery. Results showed that the HIIT program resulted in significant reductions in subcutaneous fat (1.6\%) and body weight (3.9 $\mathrm{kg}$ ). There is also evidence to examine the efficacy of HIIT programs in athletes (24-27) with regard to body composition. In line with our results, Balbasi et al. (24) observed a decrease in body weight and thickness of body fat of futsal players after a 3-week running-based HIIT program. In another study, Hottenrott et al. (25) reported that 4-30 min sessions of HIIT and an additional 30 min endurance run weekly lead to improve the body mass in recreational endurance runners. These authors explained that the main reason for reduction of total body mass was a $16.5 \%$ reduction of visceral fat. Although we did not measure visceral fat in the present study, it was proposed that HIIT may be more favorable for improvements of body composition in terms of visceral fat (25).

However, some other studies have shown that HIIT protocols do not improve body composition in elite swimmers (27) and judo athletes (26). The inconsistency between results may partially account for the type of athletes (i.e., wrestlers vs. swimmers) and different HIIT protocols. For example, Franchini et al. (26) used lower-body and upper-body cycle-ergometer in two different groups, and a specific group which was performed two blocks of 10 sets of $20 \mathrm{~s}$ of a single judo technique entrance. In the present study, the HIIT protocol included 8 wrestling techniques that were performed in 2-8 bouts throughout the study; while in the aforementioned study (26), only one judo technique was performed. Thus, higher training volume can be considered as a reason for these apparent discrepancies.

Although we observed a significant increase in AG after the study protocol in all the groups, but AG was significantly higher in HIIT groups (approximately twofold) compared to control group. The findings of the present study were similar to those reported by Fathi et al. (28), namely that a 12-week high-intensity exercise program consisting of treadmill running raised fasting AG levels. In support of this finding, Leidy et al. (29) investigated whether a 3-month body weight reduction program in normalweight, healthy women that consisted of exercise and energy deficit-imposing diet affected AG concentrations. The exercise intervention led to increase AG levels only in the weight-loss group and changes in ghrelin were negatively correlated with changes in body weight (29). Thus, it seems that chronic exercise produces increases in AG levels, especially when weight loss is occurred.

Ghrelin can affect all aspects of the energy homeostasis system in a concerted manner that contributes to promote weight gain. If ghrelin is an adiposity-associated hormone, its concentrations should also show compensatory changes in response to changes in body weight, specifically, increasing with weight loss and decreasing with weight gain (30). Since exercise itself does not directly influence AG levels $(29,30)$, and the amount of energy intake did not differ between the study groups, it can be stated that increased AG concentrations which is 
observed in the present study, has been resulted from HIIT-induced weight loss.

\section{CONCLUSION}

Generally, we conclude that eight weeks of wrestling-based HIIT can lead to significant improvements in body composition, specifically, body fat percentage and weight which is related to increase in total levels of plasma AG in highly trained wrestlers.

\section{APPLICABLE REMARKS}

- It is suggested that wrestling-based HIIT without any change in energy intake can be used to manage body weight and decrease body fat percentage in youth wrestlers.

\section{REFERENCES}

1. Hall KD, Heymsfield SB, Kemnitz JW, Klein S, Schoeller DA, Speakman JR. Energy balance and its components: implications for body weight regulation. Am J Clin Nutr. 2012;95(4):989-94. [DOI:10.3945/ajcn.112.036350] [PMID] [PMCID]

2. Islam H. The Effects of Exercise Intensity on Acylated Ghrelin, Active Glucagon-like Peptide-1, and Appetite: Examining The Potential Involvement of Interleukin-6. 2016:Theses and Dissertations (Comprehensive). 1851.

3. Gibala MJ, Gillen JB, Percival ME. Physiological and health-related adaptations to low-volume interval training: influences of nutrition and sex. Sports Med. 2014;44(2):S127-37. [DOI:10.1007/s40279-014-0259-6] [PMID] [PMCID]

4. Deighton K, Karra E, Batterham RL, Stensel DJ. Appetite, energy intake, and PYY3-36 responses to energymatched continuous exercise and submaximal high-intensity exercise. Appl Physiol Nutr Metab. 2013;38(9):94752. [DOI:10.1139/apnm-2012-0484] [PMID]

5. Sim AY, Wallman K, Fairchild T, Guelfi K. High-intensity intermittent exercise attenuates ad-libitum energy intake. Int J Obes. 2013;38(3):1-6.

6. Thivel D, Isacco L, Montaurier C, Boirie Y, Duché P, Morio B. The 24-h energy intake of obese adolescents is spontaneously reduced after intensive exercise: a randomized controlled trial in calorimetric chambers. PLoS One. 2012;7(1):e29840. [DOI:10.1371/journal.pone.0029840] [PMID] [PMCID]

7. Hazell TJ, Islam H, Townsend LK, Schmale MS, Copeland JL. Effects of exercise intensity on plasma concentrations of appetite-regulating hormones: Potential mechanisms. Appetite. 2016;98:80-8. [DOI:10.1016/j.appet.2015.12.016] [PMID]

8. Hazell TJ, Hamilton CD, Olver TD, Lemon PW. Running sprint interval training induces fat loss in women. Appl Physiol Nutr Metab. 2014;39(8):944-50. [DOI:10.1139/apnm-2013-0503] [PMID]

9. Heydari M, Freund J, Boutcher SH. The effect of high-intensity intermittent exercise on body composition of overweight young males. J Obes. 2012;2012(12):1-8. [DOI:10.1155/2012/480467] [PMID] [PMCID]

10. Skelly LE, Andrews PC, Gillen JB, Martin BJ, Percival ME, Gibala MJ. High-intensity interval exercise induces 24-h energy expenditure similar to traditional endurance exercise despite reduced time commitment. Appl Physiol Nutr Metab. 2014;39(7):845-8. [DOI:10.1139/apnm-2013-0562] [PMID]

11. Hazell TJ, Olver TD, Hamilton CD, Lemon PW. Two minutes of sprint-interval exercise elicits 24-hr oxygen consumption similar to that of $30 \mathrm{~min}$ of continuous endurance exercise. Int J Sport Nutr Exerc Metab. 2012;22(4):276-83. [DOI:10.1123/ijsnem.22.4.276] [PMID]

12. Alkahtani SA, Byrne NM, Hills AP, King NA. Interval training intensity affects energy intake compensation in obese men. Int J Sport Nutr Exerc Metab. 2014;24(6):595-604. [DOI:10.1123/ijsnem.2013-0032] [PMID]

13. Deighton K, Barry R, Connon CE, Stensel DJ. Appetite, gut hormone and energy intake responses to low volume sprint interval and traditional endurance exercise. Eur J Appl Physiol. 2013;113(5):1147-56. [DOI:10.1007/s00421-012-2535-1] [PMID]

14. King JA, Wasse LK, Stensel DJ, Nimmo MA. Exercise and ghrelin. A narrative overview of research. Appetite. 2013;68:83-91. [DOI:10.1016/j.appet.2013.04.018] [PMID]

15. Martins C, Stensvold D, Finlayson G, Holst J, Wisloff U, Kulseng B, et al. Effect of moderate-and high-intensity acute exercise on appetite in obese individuals. Med Sci Sports Exerc. 2015;47(1):40-8. [DOI:10.1249/MSS.0000000000000372] [PMID]

16. Martins C, Aschehoug I, Ludviksen M, Holst J, Finlayson G, Wisloff U, et al. High-intensity interval training, appetite, and reward value of food in the obese. Med Sci Sports Exerc. 2017;49(9):1851-8. [DOI:10.1249/MSS.0000000000001296] [PMID] 
17. Sim AY, Wallman KE, Fairchild TJ, Guelfi KJ. Effects of high-intensity intermittent exercise training on appetite regulation. Med Sci Sports Exerc. 2015;47(11):2441-9. [DOI:10.1249/MSS.0000000000000687] [PMID]

18. Rashidlamir A, Saadatnia A. The Effect of Eight Weeks of Wrestling and Wrestling Technique Based Circuit Trainingon Lymphocyte ABCA1 Gene Expression and Plasma Apolipoprotein AI. World J Sports Sci. 2011;4(2):144-50.

19. Wewege M, van den Berg R, Ward R, Keech A. The effects of high-intensity interval training vs. moderateintensity continuous training on body composition in overweight and obese adults: a systematic review and metaanalysis. Obes Rev. 2017;18(6):635-46. [DOI:10.1111/obr.12532] [PMID]

20. Khammassi M, Ouerghi N, Hadj-Taieb S, Feki M, Thivel D, Bouassida A. Impact of a 12-week high-intensity interval training without caloric restriction on body composition and lipid profile in sedentary healthy overweight/obese youth. J Exerc Rehabil. 2018;14(1):118-25. [DOI:10.12965/jer.1835124.562] [PMID] [PMCID]

21. Martins C, Kazakova I, Ludviksen M, Mehus I, Wisloff U, Kulseng B, et al. High-intensity interval training and isocaloric moderate-intensity continuous training result in similar improvements in body composition and fitness in obese individuals. Int J Sport Nutr Exerc Metab. 2016;26(3):197-204. [DOI:10.1123/ijsnem.2015-0078] [PMID]

22. Fisher G, Brown AW, Brown MMB, Alcorn A, Noles C, Winwood L, et al. High intensity interval-vs moderate intensity-training for improving cardiometabolic health in overweight or obese males: a randomized controlled trial. PloS one. 2015;10(10):e0138853. [DOI:10.1371/journal.pone.0138853] [PMID] [PMCID]

23. Keating SE, Machan EA, O'Connor HT, Gerofi JA, Sainsbury A, Caterson ID, et al. Continuous exercise but not high intensity interval training improves fat distribution in overweight adults. J Obes. 2014;2014:1-12. [DOI:10.1155/2014/834865] [PMID] [PMCID]

24. Balbasi F, Shabani R, Nazari M. Effect of high-intensity interval training on body composition and bioenergetic indices in boys-futsal players. Phys educ stud. 2016;20(5):42-8. [DOI:10.15561/20755279.2016.0506]

25. Hottenrott K, Ludyga S, Schulze S. Effects of high intensity training and continuous endurance training on aerobic capacity and body composition in recreationally active runners. J Sports Sci Med. 2012;11(3):483-88. [PMID] [PMCID]

26. Franchini E, Julio UF, Panissa VL, Lira FS, Gerosa-Neto J, Branco BH. High-intensity intermittent training positively affects aerobic and anaerobic performance in judo athletes independently of exercise mode. Front Physiol. 2016;7:268. [DOI:10.3389/fphys.2016.00268] [PMID] [PMCID]

27. Kilen A, Larsson TH, Jørgensen M, Johansen L, Jørgensen S, Nordsborg NB. Effects of 12 weeks high-intensity \& reduced-volume training in elite athletes. PloS one. 2014;9(4):e95025. [DOI:10.1371/journal.pone.0095025] [PMID] [PMCID]

28. Fathi R, Ghanbari-Niaki A, Kraemer RR, Talebi-Garakani E, Saghebjoo M. The effect of exercise intensity on plasma and tissue acyl ghrelin concentrations in fasted rats. Regul Pept. 2010;165(2-3):133-7. [DOI:10.1016/j.regpep.2010.05.013] [PMID]

29. Leidy H, Gardner J, Frye B, Snook M, Schuchert M, Richard E, et al. Circulating ghrelin is sensitive to changes in body weight during a diet and exercise program in normal-weight young women. J Clin Endocrinol Metab. 2004;89(6):2659-64. [DOI:10.1210/jc.2003-031471] [PMID]

30. Cummings DE. Ghrelin and the short-and long-term regulation of appetite and body weight. Physiol Behav. 2006;89(1):71-84. [DOI:10.1016/j.physbeh.2006.05.022] [PMID] 